\section{Ingeniería y Estado: la red nacional de caminos y las obras públicas en la Argentina, 1930-1943}

\section{Engineering and the State: the national road network and public works in Argentina, 1930-1943}

Anahi Ballent

Profesora de la Universidad Nacional de Quilmes; Investigadora del Consejo Nacional de Investigaciones Científicas y Técnicas (Conicet) Gallo $1518,3^{\text {er }}$ piso, depto. 11

1425 Ciudad Autónoma de Buenos Aires - Argentina aballent@unq.edu.ar
BALLENT, Anahi. Ingeniería y Estado: la red nacional de caminos y las obras públicas en la Argentina, 1930-1943. História, Ciências, Saúde - Manguinhos, Rio de Janeiro, v.15, n.3, p.827-847, jul.-set. 2008.

\section{Resumen}

Analiza la acción de la Dirección Nacional de Vialidad (DNV) dentro del Ministerio de Obras Públicas de Argentina al inicio de su desarrollo, entre 1932 y 1943, enfatizando la formación de una burocracia técnica de ingenieros y las distintas representaciones, técnicas y políticas, construidas alrededor del ingeniero. La hipótesis considera que la constitución de la DNV marcó un momento culminante y un punto de inflexión en la injerencia de la ingeniería como campo científico y profesional en la estructura estatal de las obras públicas, dentro de una estrecha relación entre ingeniería y estado desarrollada desde las últimas décadas del siglo XIX.

Palabras clave: historia; ingeniería; obras públicas; burocracia; Argentina.

\section{Abstract}

The article analyzes the work of the Argentinean Public Works Ministry's National Road Department (Dirección Nacional de Vialidad) during its earliest years, from 1932 to 1943, with a focus on formation of a technical bureaucracy composed of engineers and on the technical and political representations constructed around the field of engineering. The hypothesis is that creation of the Department represented both a culmination and a turning point in engineering's role as a scientific and professional field in the government public works structure, within the framework of the close ties between engineering and the state that had developed from the final decades of the nineteenth century.

Keywords: history; engineering; public works; bureaucracy; Argentina. 
$\mathrm{E}$ n mayo de 1939, el senador riojano Héctor González Iramain presentaba un proyecto de ley sobre construcción de hoteles de turismo en las provincias de La Rioja y Catamarca, que se sancionaría más tarde como ley 12.669. Ligaba su proyecto al desarrollo de la infraestructura de transporte, sobre todo de los caminos, que el Estado nacional estimulaba con particular intensidad desde octubre de 1932, momento en el cual la ley 11.658 (llamada Ley de Vialidad) creaba la Dirección Nacional de Vialidad (DNV) del Ministerio de Obras Públicas (MOP):

Los modernos medios de comunicación; el coche motor incorporado al servicio ferroviario regular; el camino duro y cuidado, para el automóvil; el ómnibus y el camión, han acercado las provincias del interior a la Capital y a todo el litoral argentino. El territorio del país se ha achicado, podría decirse, y los hombres y las cosas circulan en toda su vasta extensión, con facilidad inusitada, impulsando un comercio activo, haciendo cada día más efectiva y cierta la unidad nacional, y creando nuevas necesidades no previstas, y que ahora es imperioso contemplar y solucionar (Argentina, 16 mayo 1939, p.65).

En un país de grandes dimensiones, donde la construcción de la infraestructura de transporte resultaba particularmente compleja y dificultosa, el mensaje del senador resultaba excesivamente optimista, y, en rigor, no puede ser leído de manera literal ni considerarse descriptivo de la efectiva transformación del territorio nacional. Antes que documentar una realidad, González Iramain expresaba un viejo anhelo - el de unidad e integración nacional - y consideraba que una serie de acciones estatales del momento apuntaban en la dirección de su futuro cumplimiento. La representación proyectaba acciones reales; las excedía al mismo tiempo que se anclaba en ellas. En efecto, en 1931, poco antes de la sanción de la Ley de Vialidad, el país contaba con unos escasos $2.000 \mathrm{~km}$ de caminos de tránsito permanente, mientras que 1944 la acción pública había logrado elevar esa cifra por arriba de los 30.000km. En poco más de una década, entonces, la labor estatal consolidaría para el tránsito permanente aproximadamente un sesenta por ciento de la longitud en que se estimaba la red nacional de caminos. Uno de los primeros datos que deben evaluarse para considerar la importancia que se asignaba al sector es la cantidad de automotores en circulación en el país, ya que pese a las dificultades de importación de unidades como consecuencia de las políticas cambiarias posteriores a la crisis de 1929, dicha cantidad (405.743) ubicaba a Argentina en el séptimo lugar mundial, superando, en el caso de naciones americanas, a Brasil (160.000) y a México (99.470) (Argentina, 1939, p.111).

El extendido optimismo frente al avance del camino que aquí se presenta, ejemplificado en el discurso de un político de una provincia norteña, actualizaba un anhelo decimonónico argentino, aquel de constituir un país moderno e integrado que ya había impulsado el trazado de las líneas de ferrocarriles en el siglo XIX. ¿En qué sentido era nueva, entonces, la aspiración de control del territorio argentino materializada en los años 1930? En primer lugar, en que, a diferencia de los ferrocarriles, explotados en gran medida por empresas privadas, el sistema de caminos era pensado de manera integral desde el Estado; en segundo lugar, en que, al menos en teoría, abría posibilidades de desplazamientos más libres y abiertos que los permitidos por el ferrocarril; y, en tercer lugar, por la forma en que los medios de comunicación insistieron en este tema, asignándole una particular presencia dentro de la sociedad a través de la prensa escrita, la radio, también 
el cine y, en muchos casos, a través del deporte. Finalmente, la estructura de la acción estatal en vialidad, de planificación centralizada, pero con divisiones operativas regionales en todo el país, coordinada además con las direcciones de vialidad provinciales, le daba a los protagonistas y a sus obras una presencia extendida a través del territorio que alcanzó niveles notables: los 'ingenieros de vialidad' y sus técnicos se incorporaron al elenco de representantes del Estado con fuerte presencia en la sociedad.

Todos estos elementos sustentaron también el carácter de disparador de expectativas colectivas que adquirió la red de caminos. Al mismo tiempo, tal carácter encontró bases en la cantidad de actores y fuerzas sociales que se movilizaron a su alrededor y en el grado de dispersión social que tales actores registraban. Nos referimos al Estado y a los ingenieros, a la industria del automotor - no solo los importadores, las compañías extranjeras o empresas productoras de nafta sino también un mundo local mucho más amplio y popular, el de los mecánicos, los carroceros y la incipiente industria nacional autopartista, a las asociaciones de automovilistas y promotores del turismo, a los deportistas del automóvil y su público. De manera más abarcadora, puede afirmarse que la fuerza del par automóvil-camino, como disparador de un imaginario social, se basó en una concurrencia de factores: intereses económicos y sociales amplios y dispersos, transformaciones materiales y amplificación y recreación mediática de las mismas. La política vial, entonces, y sobre todo en los años 1930, momento de su consolidación dentro del Estado, ostentaba una capacidad de condensación de expectativas sociales que no todas las políticas de transformación territorial eran capaces de generar.

Aunque formaba parte de una historia nacional más extensa, la política vial se vinculó directamente con ciertas condiciones políticas y económicas de los años 1930. En el plano político, el período se caracterizó por la relativa continuidad de lo que de manera rápida, aunque no del todo precisa, puede definirse como el 'auge conservador'. Marcado inicialmente por el golpe militar que en 1930 destituyó al presidente Hipólito Yrigoyen, líder del llamado sector personalista de la Unión Cívica Radical, en su segundo mandato presidencial, el período estuvo dominado a partir de 1932 por la Concordancia como fuerza política (coalición de radicales antipersonalistas, socialistas independientes y fuerzas conservadoras provinciales) y registró inicialmente el gobierno de facto de José F. Uriburu y luego las presidencias constitucionales de Agustín P. Justo (1932-1938), de Roberto M. Ortiz (1938-1942) y de Ramón Castillo (1942-1943). El período puede considerarse finalizado a partir del golpe militar de junio de 1943, que inauguró nuevos desarrollos políticos y sociales que desembocaron en los dos gobiernos peronistas (1946-1955) (Macor, 2001). En el plano económico, el período se encontró marcado por los efectos adversos de la crisis económica mundial desatada en 1929, que golpeó fuertemente a una economía que, desde las últimas décadas del siglo XIX, había crecido en base a la exportación de su producción agropecuaria. En los años 1930 se registró un incremento de la ingerencia estatal en la economía, cuyos objetivos primarios consistirían en contrarrestar los efectos de la crisis y de la inestabilidad de los mercados internacionales. Al mismo tiempo, las nuevas condiciones de intercambio aconsejaron limitar las importaciones, introduciendo medidas proteccionistas que estimularon un desarrollo industrial intensificado a partir de 1939, al estallar la guerra europea. Como consecuencia de estas medidas, aunque no siempre en 
total acuerdo con la intención de los actores que las promovían, el principal motor de la economía se desplazó entre 1930 y 1950 de las actividades agrícolas ganaderas destinadas a los mercados externos, a las actividades industriales dirigidas al consumo interno (Korol, 2001). Estos procesos encontraron un soporte en una ampliación del aparato estatal que no solo incluyó nuevas instituciones reguladoras de ciertas actividades económicas (como las juntas reguladoras de la producción de carne o de vinos, entre otras), sino que amplió hacia nuevos campos la tradicional función del Estado como proveedor de la infraestructura física requerida por las actividades económicas a través de las obras públicas.

Dentro de este marco, distintos aspectos de la política vial del período han sido objeto de estudio, sobre todo desde perspectivas de la historia económica. Así, se ha analizado su inserción en las políticas económicas de los gobiernos conservadores, su relación con los fuertes avances de la industria automotriz norteamericana en América Latina desde fines de los años 1910 y su conflictiva relación con los ferrocarriles británicos que habían monopolizado las redes públicas de transporte desde las últimas décadas del siglo XIX (García Heras, 1985), tanto como la influencia de los importadores de automóviles en diversas asociaciones que apoyaban el desarrollo vial (Ospital, 2002). También se han registrado aportes de la historia social, por ejemplo en la relación entre automóvil, camino y expansión del turismo (Scarzanella, 1998), y la ingerencia de algunas entidades públicas no estatales, como el Automóvil Club Argentino (ACA) y el Touring Club en la construcción de políticas de obras públicas, por un lado, y en la extensión de la cultura del automóvil, por otro (Piglia, 2005, 2007).

Otro conjunto de trabajos, guiados por el interés de las transformaciones del territorio por parte del Estado y las representaciones sociales que acompañaron dichas transformaciones, han tratado de analizar su acción en términos culturales, dentro del marco de un período histórico caracterizado por un incremento de la presencia del Estado en la economía y la sociedad (Gorelik, 1987; Ballent et al., 2001; Torrent, 2001). En el mismo sentido, se ha analizado la trama de actores sociales que impulsaron el avance de la construcción vial en relación con la construcción de un universo de representaciones del territorio centrado en el camino (Ballent, 2004, 2005a). Menos atención se ha prestado específicamente a los diseñadores de estas políticas, en su doble carácter político y técnico: la acción de los ingenieros viales actuando en las nuevas reparticiones estatales.

Esta perspectiva propone cruzar una historia del Estado con una historia de las disciplinas científicas y de las formas de ejercicio profesional. Se trata de dos campos de poco desarrollo en el caso de la ingeniería argentina, aunque existen algunos episodios estudiados y temas, afines a los que se proponen aquí, con énfasis en la historia económica (Salerno, 2003; Palermo, 2005) o institucional (Ballent et al., 2004) que no pueden sino recortarse sobre la más reciente historia abarcadora de la ingeniería en Argentina (Lucchini, 1981), como lo hacen otros estudios que indagan en aspectos específicos o ramas de la ingeniería (Lobato, ago.-dic. 1998; Silvestri, 2003). Teniendo en cuenta el estado de los estudios, este trabajo se propone como una contribución preliminar que, lejos de agotar el tema, señale su potencialidad y sus dimensiones de interés.

La hipótesis de este artículo considera que la constitución de la DNV en los años 1930 marcó un momento culminante pero también un punto de inflexión en la injerencia de 
la ingeniería como campo científico y profesional en la estructura estatal de las obras públicas. En tal sentido, el nuevo organismo señaló un punto máximo de relación entre dicho campo y el Estado, relación estrecha que contaba con una historia previa desde las últimas décadas del siglo XIX; pero al mismo tiempo dicho organismo permite registrar claros signos de transformación del vínculo que se observan en el interés de los ingenieros en la diversificación de las formas de vinculación con el Estado, en el avance científico de nuevas ramas de la ingeniería más ligadas a la actividad privada que a la estatal y en la expansión de las formas de ejercicio privado de la profesión.

El argumento afirma que las políticas de obras públicas de los años 1930 deben ser analizadas en función de sus condicionantes económicos y sociales, puntos que ya han sido encarados en trabajos anteriores, pero también en relación con aspectos específicamente políticos de la época que estuvo marcada por el golpe militar que en 1930 destituyó a Yrigoyen y por la conformación de una burocracia técnica desde fines del siglo XIX, la de los ingenieros actuando en el interior de la estructura estatal. En tal sentido, de manera similar a la que hemos presentado en esta introducción, el camino (como sinécdoque de la política vial) será pensado como disparador de imágenes y representaciones, pero no serán ya representaciones de la relación camino-sociedad, como las indicadas anteriormente, sino representaciones de la obra pública y de la ingeniería proyectadas hacia los campos del Estado, del gobierno y de la política dentro del particular marco constituido por el aparato estatal del período 1930-1943.

\section{El marco histórico de las obras públicas: el Ministerio de Obras Públicas y su burocracia técnica}

La DNV no fundó las características que se han indicado en el punto anterior, sino que las actualizó y, en algunos casos, las exasperó dentro de las oportunidades brindadas por las particulares coordinadas políticas de los años 1930. Pero en rigor, ellas pueden rastrearse en la historia de las obras públicas, a partir del momento de institucionalización que supuso la creación del MOP en 1898. Se trató de un momento clave en la construcción del aparato estatal cuyas funciones y dependencias se expandían, se ordenaban y especializaban (Oszlak, 1997). En particular, para los estudios sobre el Estado en Argentina, el caso del MOP es particularmente rico, porque, dentro del marco de las conocidas discontinuidades que caracterizan la historia política e institucional argentina, el organismo registra una estabilidad notable que sostuvo su homogeneidad por unos cincuenta años y su desarrollo completo por una centuria, ya que perdió su rango institucional recién en los años 1990. En un Estado sumamente dependiente de los cambios políticos y muy permeado por las demandas y presiones de la sociedad, como es el argentino, alejado de modelos más estables y autónomos, como los modelos trazados por los análisis del Estado de Max Weber, el MOP presenta una burocracia técnica de un nivel de consolidación fuerte y de un grado de autonomía que, al menos hasta el período de estudio de este trabajo no parece haber sido desdeñable. Para la década de 1930 se trataba de un organismo consolidado: el rascacielos modernista proyectado por la Dirección de Arquitectura para su propio funcionamiento e inaugurado en 1936, que albergaba a los dos mil empleados de su estructura centralizada, 
constituía un símbolo de un poderoso organismo en expansión. La DNV, entonces, resultó heredera de esta historia y explotó sus características como repartición ejemplarizadora dentro del particular contexto político de los años 1930.

Siguiendo el modelo francés, el MOP se originó como una estructura centralizada, con asiento en Buenos Aires y delegaciones o secciones regionales en el interior de la República, por repartición. En cuanto a su estructura técnica se inició con cuatro direcciones generales: de Vías de Comunicación y Arquitectura, de Obras Hidráulicas, de Obras de Salubridad y de Contabilidad (Espósito, 1944). Los ferrocarriles eran entonces las obras que mostraban mayor diferenciación y complejidad interna, ya que era uno de los programas de mayor desarrollo en el momento. En las décadas siguientes, las reparticiones se fueron especializando, en parte en función de las demandas de la política y en menor medida como consecuencia del proceso de especialización que fueron sufriendo las ramas de las ingenierías. Tomaron en general la forma de direcciones generales o nacionales, es decir dependencias directamente subordinadas a la conducción centralizada. En algunos casos, por distintos motivos y asumiendo diferentes formas, se crearon reparticiones descentralizadas, con cierto grado de autonomía, cuyo sentido varió en el tiempo: la Administración General de los Ferrocarriles del Estado (1910); Obras Sanitarias de la Nación (1912) y, en el período que nos ocupa, la DNV (1932). La creación de nuevas reparticiones va indicando la aparición de nuevos temas prioritarios dentro del programa de obras públicas. Para tener una idea de la magnitud de la estructura, recordemos que para 1940, además de estas tres reparticiones descentralizadas, existían seis Direcciones Generales: de Irrigación, de Ferrocarriles, de Navegación y Puertos, de Arquitectura, de Estudios y Obras del Riachuelo y de Contabilidad. Se trata de una unidad compleja, con una inmensa diversidad interior y es posible definirla como un conglomerado integrado por unidades de distinto tipo, donde reparticiones más modernas coexisten con otras que parecen haber quedado ancladas en el pasado, como si en su interior operaran tiempos diversos. Además, el juego entre las distintas reparticiones y la valoración política y social de sus objetivos definía jerarquías entre las mismas que variaban en el tiempo.

La observación de la historia del MOP indica que entre 1898 y 1943 se desplegó el programa inicial del ministerio, ya que sus desarrollos institucionales, su crecimiento, pueden considerarse comprendidos dentro del sentido del programa inicial, diseñado y ejecutado por una burocracia de ingenieros. Las características de ese modelo inicial fueron las siguientes: operar sobre obras más ligadas a la infraestructura económica que a la social; tender a la concentración de la obra pública; basarse en una burocracia de ingenieros; adoptar una organización que tiene que ver sobre todo con la especialización de la ingeniería; operar sobre todo dentro de campos aislados - definidos como se indica en el punto anterior -, ofreciendo resistencias a la integración horizontal.

El crecimiento del organismo consolidó una burocracia técnica basada en ingenieros, aunque en el momento en que se creaba el ministerio, la ingeniería llevaba ya unos ochenta años de inserción dentro del Estado, primero en el caso de los topógrafos, luego en el de los ingenieros civiles, en una inserción que se vio particularmente estimulada a partir de la construcción de ferrocarriles y puertos de la década de 1870 en adelante. Aunque el MOP no llegó a concentrar la totalidad de la obra pública producida por el Estado, ni era la 
única repartición pública que aglutinaba una cantidad importante de ingenieros, era un sinónimo de la ingeniería nacional, que en 1870 había producido los primeros egresados argentinos de la carrera de ingeniería dentro del Departamento de Ciencias Exactas (Universidad de Buenos Aires), creado en 1866. Para 1900, las distintas carreras o escuelas nacionales que fueron formándose (la citada de Buenos Aires, la perteneciente a la Universidad de Córdoba a partir de 1879, la de la Universidad de La Plata en 1897 y la Escuela de Minas de San Juan, de 1871) habían producido 250 profesionales (Lucchini, 1981, p.119-126).

Es decir que el ministerio encauzaba y aceleraba procesos existentes: la formación de importantes asociaciones de ingenieros, como el Centro Nacional de Ingenieros - luego Centro Argentino de Ingenieros, 1896 - fue casi contemporáneo al mismo. La relación entre el MOP y las instituciones de sociabilidad y defensa de la profesión y las de enseñanza de la ingeniería y de formación de técnicos, relación presente en el momento de su creación, se mantuvo a lo largo del tiempo. En el caso de la DNV, en los años 1930, el nuevo plan de estudios de ingeniería civil de la Universidad de Buenos Aires, puesto en práctica en 1937, contempló la orientación 'Vías de comunicación', a partir del curso 'Carreteras', dictado por el ingeniero Teodoro Sánchez de Bustamante, ingeniero jefe de la DNV, mientras que en base a la misma demanda estatal, la Escuela Industrial de la Nación Otto Krause creaba un curso para preparar técnicos.

En términos más amplios, el MOP era un organismo de investigación (varias reparticiones contaban con laboratorios, talleres y cuerpos experimentales), difusión técnica y científica (a través de publicaciones especializadas) y de formación de recursos humanos en distintos niveles (contaba con diferentes tipos de planteles profesionales, técnicos y obreros). En otras palabras, ninguna empresa o actividad privada local podía sustituir la inmensa experiencia que brindaban estas reparticiones especializadas en proyectos y en algunos casos en ejecución, administración y explotación de obras de ciertas ramas de la ingeniería. El MOP, entonces, constituía una pieza clave del campo disciplinar de los ingenieros, y no sólo porque concentrara un alto número de profesionales.

En cuanto al análisis de la construcción de representaciones que acompañó la consolidación de la estructura burocrática del MOP, existe una fuente sumamente valiosa, que es el Boletín de Obras Públicas, publicado desde 1900 hasta 1945 aproximadamente (con algunas discontinuidades), dirigido a quienes pueden denominarse los actores del mundo de la obra pública: los niveles técnico-profesionales del ministerio, otras reparticiones estatales emparentadas a ese organismo por temas, profesionales, políticos interesados en la obra pública, empresarios, contratistas y proveedores del Estado. La fuente no sólo informa sobre las políticas y sobre su funcionamiento y el de otros sectores públicos afines, sino que además muestra consistentes acciones estatales para construir un cuerpo técnico estatal y también presenta la forma en que la pertenencia a la institución legitimaba a los profesionales, sobre todo a los ingenieros. Se observa allí una construcción intensa de identidades disciplinares y profesionales en relación con el Estado, una jerarquía de personajes, una valorización de las trayectorias. También se registra una fuerte confianza en el poder de los técnicos en la determinación de las políticas y un intenso orgullo en la pertenencia a este cuerpo estatal. El personal administrativo, técnico no-profesional (que 
era numeroso y en muchos casos calificado) u obrero, en cambio, casi no aparecía. En otras palabras, los ingenieros eran presentados como el núcleo vital del ministerio.

En los perfiles profesionales de ingenieros que muestran las notas del Boletín de Obras Públicas se resaltaba frecuentemente una carrera meritocrática dentro del Estado, mejor aún si se desarrollaba dentro de una misma repartición, en un sentido que favorecía la especialización. Efectivamente, ese era un camino que se había consolidado para los años 1930, cuando muchos ingenieros se jubilaban luego de haber desempeñado tareas en el MOP durante toda su vida laboral, ascendiendo desde los cargos técnicos más bajos. También se valoraba la docencia universitaria. Inserción en el Estado, carrera burocrática y enseñanza universitaria eran tres aspectos que se legitimaban mutuamente. Se destacaban las obras realizadas o proyectadas dentro de la estructura estatal, la participación en eventos científicos presentando temas de su especialidad y los numerosos viajes al exterior que solían realizar, enviados o solventados por el Estado, para realizar compras o relevar experiencias extranjeras.

Encontramos ciertas regularidades, entonces, en la forma en que la publicación del MOP presentaba a estos profesionales, pero esas mismas regularidades se observan también en las biografías escritas por ingenieros (Besio Moreno, 1945, por ejemplo) o en las historias narradas en la revista La Ingeniería por el Centro Argentino de Ingenieros. Es decir que los órganos corporativos compartían el carácter prestigioso de estas representaciones. En esas historias no se hacía hincapié, en cambio, en las 'entradas' y 'salidas' de la estructura estatal, tampoco en la participación de los ingenieros en empresas privadas, ni en el rol de empresarios que muchos de ellos desarrollaron. Estos son aspectos que se encuentran soslayados y cuya consideración complejizaba el ejercicio real de la profesión y el perfil de sus actores. Si se comparan los datos biográficos presentados por publicaciones estatales o corporativas con los que aparecen en Quien es quien, o con Personalidades de la Argentina, la imagen pública que proyectan los ingenieros cambia. Sobre este tema volveremos posteriormente. Lo importante en este punto del análisis es la constatación de su existencia como representación.

\section{La creación de la Dirección Nacional de Vialidad y la reforma del Estado}

Dentro del marco general referido al MOP, ubicaremos ahora la acción de la DNV en los años 1930, para observar el rol que cumplió. Recordemos previamente que, aunque la demanda a favor de una mayor ingerencia estatal en la vialidad era un reclamo que distintos actores reiteraban sobre todo desde el fin de la Primera Guerra Mundial, la reacción a la crisis económica de 1930 fue la circunstancia que, desde el punto de vista de la política gobernante, mostró como particularmente oportuna la propuesta. Dentro de una demanda amplia, los ingenieros en particular habían incorporado cursos de Caminos en las carreras de ingeniería civil desde la primera década del siglo, posiblemente en relación con la ley 5315/07 o Ley Mitre que fomentaba la construcción de caminos de acceso a las estaciones ferroviarias. También insistían en la relevancia del tema frente al creciente avance automotor en importantes eventos del campo disciplinar, como los congresos nacionales de ingeniería, iniciados en 1916, y los congresos nacionales de vialidad, organizados por el Touring Club desde 1922 (Lucchini, 1981). 
Pese a las presiones, la acción sistemática y de magnitud por parte del Estado fue encarada recién en la década de 1930. Como otras propuestas legislativas presentadas aproximadamente al mismo tiempo, el proyecto oficial de 1932 hacía hincapié en el abaratamiento del flete como una forma de reducir los costos de la producción rural, con el objeto de mejorar la competitividad de los productos argentinos en el mercado internacional. En este sentido podemos considerar que la Ley de Vialidad formaba una pareja con otra ley sancionada muy poco después, la 11.742 de elevadores de granos, nueva iniciativa oficial en el campo de las obras públicas, presentada bajo la presión de la crisis y centrada también en la modernización del comercio de la producción agrícola. A este objetivo central se sumaban otros, como el de combatir la desocupación a través de la obra pública, pero de manera subordinada a la primacía asignada al rol económico del camino en relación con la producción agraria. Sólo más tarde y recién en la medida en que las consecuencias de la crisis económica se fueron superando, se sumarían nuevos objetivos como el estímulo al turismo, posterior a la sanción de la ley de Parques Nacionales en 1934 o a ciertas obras de vialidad urbana a partir de 1937, cuando la DNV, al recibir el encargo de ejecución de la avenida de circunvalación de Buenos Aires (avenida General Paz), creó su División Accesos a las Grandes Ciudades.

Como se indicó anteriormente, la ley 11.658/1932 creó un nuevo fondo nacional de vialidad destinado al estudio, trazado y construcción de caminos y obras anexas, obtenido por un impuesto sobre la nafta y los lubricantes. Desde el punto de vista institucional, la Dirección General de Vialidad de la Nación, dependiente del MOP, creada unos meses antes de la sanción de la ley, sobre la base de dos reparticiones existentes, fue ascendida a Dirección Nacional (DNV) con la misión de la planificación y construcción de la red vial troncal o nacional. La ley estableció también una "ayuda federal" que se repartía entre las provincias para que aumentaran sus propias inversiones en caminos provinciales, creando sus propias direcciones provinciales de vialidad (Argentina, 1938).

El primer director de la nueva repartición, que se desempeñó durante la presidencia de Justo, entre 1932 y 1938, fue Justiniano Allende Posse, ingeniero civil y empresario cordobés, figura también ligada a la política. Era director de una de las principales fábricas de cemento (Corcemar), había sido ministro de Obras Públicas de Tucumán y de Córdoba por breves períodos al inicio de la década (Diccionario..., 1948). Integraba del círculo más estrecho de colaboradores del presidente Justo, recordemos, también ingeniero civil. El flamante directivo inició acciones de inmediato: "Haremos el máximo esfuerzo para que la red de carreteras sea pronto una realidad que singularice y prestigie a este gobierno", proponía al iniciar sus funciones (Argentina, 1932, p.22). Al menos en el plano de las representaciones sociales referidas a la labor estatal, lograría su propósito: la obra inicial de la DNV fue considerada de manera muy amplia uno de los aciertos de la presidencia de Justo (él mismo militar a la vez que ingeniero civil) y por extensión, de los gobiernos conservadores del período.

La nueva repartición, entonces, se propuso trazar el plan de carreteras troncales, abarcando la totalidad del territorio con una visión unitaria y sistemática. Este objetivo, junto al privilegio del transporte de la producción, impulsaron la búsqueda de transitabilidad de las rutas y la ejecución de los caminos de costo intermedio y de bajo costo, siguiendo el modelo de los Estados Unidos, país al cual los funcionarios y técnicos viajaban 
frecuentemente, manteniendo fluidos contactos con sus colegas del Norte. La DNV traducía y publicaba numerosos trabajos técnicos que difundían la literatura norteamericana en el tema. Al mismo tiempo, el prestigio del modelo norteamericano venía sostenido también por una serie de vínculos previos, entre técnicos y políticos, que tiñeron en gran medida el desarrollo de la vialidad en América Latina y que pueden emblematizarse en el anhelo colectivo de materialización de la Ruta Panamericana. Desde 1925, cuando se realizó una primera reunión en Buenos Aires, promovida por la Unión Panamericana, comenzaron a funcionar con carácter permanente los congresos panamericanos de carreteras, creando una trama de técnicos y funcionarios de los distintos países integrantes de la unión, en la cual los ingenieros y las obras norteamericanas constituían la vanguardia del sector. Sumando estos datos al interés en la promoción de los caminos manifestado por la industria automotriz norteamericana, en un momento en que se imponía sobre las europeas en la penetración en América Latina, no sorprende la vigencia de Estados Unidos como referencia para la acción (García Heras, 1985; Barbero et al., 2003). Estos eran los condicionantes que operaban específicamente en el caso de la ingeniería vial; pero, cabe destacar, que en otros temas o ramas de la ingeniería que tomaban fuerza en la entre guerra (ingeniería industrial, en petróleo, en generación de energía hidroeléctrica, entre otras), las referencias norteamericanas desplazaban a la hegemonía de los ejemplos y figuras europeas que habían caracterizado el siglo XIX.

Pero también es necesario considerar que, aunque Estados Unidos constituía una referencia obligada, no se imponía mecánicamente, sino que los técnicos y funcionarios locales la interpretaban, seleccionando sus datos en función de los intereses y necesidades propias, como por ejemplo, para el caso de la legislación, las afinidades de organización política entre los dos países. Allende Posse justificaba la referencia en cuanto al tipo de caminos empleado en su evaluación de las necesidades argentinas: "Siempre he creído necesario construir una red extensa de caminos en vez de unos pocos de alto costo y esa opinión ha alcanzado la fuerza de una convicción al viajar por aquel país (los Estados Unidos) y al examinar una vasta extensión de su red y los mapas y planillas demostrativas del conjunto" (Argentina, 1936, p.9).

Con respecto a los materiales de construcción de los caminos, ante la disyuntiva de la época entre el uso de cemento y de asfalto, la labor de la DNV optó tempranamente por el primero considerando que se trataba de una materia prima de importante producción nacional, que no exigía importaciones ni operadores extranjeros. Al mismo tiempo, se siguió el modelo norteamericano para estimular la experimentación en materiales de bajo costo y de neta producción local (Los materiales ..., mayo 1933). Así lo anunciaba el presidente de la DNV: "Es menester que se nos hagan familiares una serie de vocablos ingleses casi intraducibles como 'loam', 'gumbo', etc.; debemos saber que óptimos materiales para caminos, como el 'top-soil' o el 'sand-clay', son una mezcla íntima de arena con un poco de arcilla que obra a manera de mortero" (Argentina, 1934a, p.8). Allende Posse, entonces, tomaba como modelo el Estados Unidos rural, al territorio americano rústico antes que al urbano, pese a que este último también se encontraba abocado al mejoramiento de las vías de comunicación, rápidas y de alto costo.

No sólo en este aspecto la propuesta del Estado argentino delineaba lo que puede denominarse una modernización conservadora, definida como una aplicación de nuevas 
técnicas o tecnologías en un sentido transformador, que en términos políticos y económicos buscaba mantener los centros tradicionales de la economía y la sociedad. En efecto, según las instrucciones de la DNV, las obras de vialidad no debían competir por la mano de obra con las producciones primarias locales en momentos de cosecha, recolección o vendimia; por el contrario, se debía coordinar el trabajo agrícola con el vial (Argentina, 1934b, p.4243). Según la política que conducía el Estado, lo central para el país, aunque la crisis de 1930 pusiera en duda sus posibilidades futuras, era la economía agraria, que por lo tanto debía ser preservada de la competencia del crecimiento industrial, complementario con respecto a la primera.

Los mandatos de esta modernización conservadora no siempre coincidían con lo que sectores de la sociedad esperaban de los caminos, tal como numerosos conflictos lo indicaban. Los ingenieros viales eran los encargados de discutir las demandas sociales que esta perspectiva consideraba equivocadas, como se observa en las palabras de Enrique Humet, ingeniero jefe de la Sección Caminos de Costo Intermedio y Bajo Costo de la DNV:

\begin{abstract}
Nuestro público interviene de buena fe en muchas de nuestras cuestiones viales, pero con frecuencia equivocadamente. Un trazado que favorezca algunos intereses particulares o locales, aunque sea en perjuicio de otras regiones o localidades, origina firmas, pedidos, entrevistas, reuniones y declaraciones ... . Un camino de tierra, para muchos peticionantes, debe convertirse en un camino de alto costo, de los tipos más costosos y con ancho de boulevares. Olvidan que hay una relación entre el tráfico y el tipo y ancho de pavimento y que excederla lesiona los intereses del país ... . El primer principio que como una bandera debería cubrir nuestra ingeniería vial de esta primera etapa proviene del camino como entidad económica ... : Ningún camino se mejorará en exceso a su capacidad de restitución. La economía de trasporte es la única medida de las mejoras a realizarse. Opuestamente, si dejamos que bellas palabras como: caminos de fomento, turismo, defensa nacional, beneficio general y confort intervengan decididamente en la distribución de los fondos de los caminos, en la elección de los tipos de obras y en el orden de las construcciones, imperará una anarquía de criterio que hará posible y justificable la intervención del público con su orientación equivocada a veces (Humet, jul. 1936).
\end{abstract}

Uno de los conflictos más sonados consistió en las discusiones públicas generadas por la traza y el tipo de camino a ejecutarse entre Buenos Aires y el preciado centro de veraneo de las clases altas, Mar del Plata, proyectado en 1934. Si bien el camino registraba un sentido productivo (constituía parte de la ruta Buenos Aires-Bahía Blanca), tenía también un alto interés turístico, y no pocos de sus usuarios, en tanto veraneantes, esperaban de esa vía un elevado grado de confort, carácter que según la DNV exigía una inversión desmesurada, posición que la repartición flexibilizó en parte frente a las presiones sociales, pero no abandonó por completo (Argentina, 1934b, p.81-99). Los técnicos de la DNV, los ingenieros, eran los encargados de defender este programa de racionalidad técnica y económica, persuadiendo de su carácter elevado a favor del interés general y resistiendo con firmeza los embates de la sociedad. Eran los depositarios de una razón técnica que se traducía en el beneficio general de la sociedad, en términos políticos. Tenían, por lo tanto, una inmensa responsabilidad; el discurso oficial de la repartición planteaba que había que encarar una "intensificación de propaganda capaz de introducir en la mente de cada ingeniero el concepto de que nuestra obra debe realizarse como se ejerce un apostolado, 
porque se trabaja para asegurar el progreso de los argentinos y la grandeza de la nación" (Argentina, 1935, p.20).

En la práctica, este programa inicial de la institución se vio forzado a complejizarse en años posteriores, sobre todo poco después de finalizada la gestión de Allende Posse. Ya en la gestión de Salvador Oría (1938-1940), las presiones de la política y de la sociedad, que mostraron ideas más complejas que las aceptadas por la gestión inicial, incorporaron como objetivos el fomento del turismo (aunque de manera moderada) y los accesos a las grandes ciudades. Al mismo tiempo, la propia repartición ponía en duda los beneficios de los 'caminos de bajo costo' que exigían alto mantenimiento. Sin embargo, la representación de los ingenieros viales, asociada a la lucha por mantener transitables los caminos de los confines de la patria, con economía y eficiencia, no perdió su vigencia.

En efecto, la imagen de los ingenieros capaces de ponerse al servicio del "progreso de los argentinos y la grandeza de la nación" actualizaba un tópico de larga data: el carácter patriótico de estos profesionales modernos, cuya acción se basaba en el dominio de la ciencia y de la técnica. Este tópico se renovaba a propósito de la empresa vial y la figura del ingeniero, dentro del Estado de los años 1930, representaría la contra cara de la imagen tradicional del empleado público, considerado inepto e incumplidor, poco calificado desde el punto de vista técnico, nombrado por el favor político en respuesta a una lealtad espuria. Esta imagen recurrente en los discursos de los políticos conservadores se encontraba exacerbada por el contexto político posterior al derrocamiento del gobierno radical de Yrigoyen, tema sobre el cual volveremos. Dentro de esta caracterización entraba el personal de la extinta Dirección General de Vialidad (antecedente breve de la Dirección Nacional, formado por la fusión de dos reparticiones existentes) cuyo cuerpo burocrático comprendía 655 empleados con funciones no técnicas, mientras que sólo eran 144 quienes contaban con conocimientos de tal tipo (ingenieros, dibujantes, proyectistas) (Argentina, 1933, p.27). Según las memorias de la DNV, la nueva repartición, en cambio, concentró a lo largo del período alrededor de dos mil empleados, dentro de los cuales, los de menor calificación técnica (categoría administrativa y de servicios) representaban alrededor del 30\%, mientras que el mayor porcentaje se encontraba directamente relacionado técnicamente con los proyectos y las obras: profesionales, subprofesionales y personal de inspecciones de obras. En otras palabras, los ingenieros y técnicos tenían un rol destacado dentro de la institución que la diferenciaba de otras reparticiones estatales.

Otro aspecto en el cual la nueva repartición se presentaba como moralizadora de las conductas de los empleados públicos se encontraba en el propio texto de la Ley de Vialidad. La norma limitaba el porcentaje del presupuesto que la repartición podía destinar a sueldos: el 8\% de su presupuesto. En la ceremonia inaugural de la repartición, Allende Posse explicaba las consecuencias de este límite: "La sabia imposición de la ley de reducir al 8\% el porcentaje de gastos burocráticos deberá ser cumplida inexorablemente y por ello será necesaria la ayuda y sacrificio de nuestros colaboradores" (Argentina, 1933, p.22). En el mismo discurso se indicaba la forma en que ese dato era considerado parte de una reforma del Estado en relación con su personal:

Debemos aplicar métodos nuevos, en parte previstos por la ley: concursos, ascensos, escalafón, estabilidad del personal, todo lo cual exige condiciones especiales de competencia, apartarse 
de la rutina y que cada funcionario, cada modesto empleado, comprenda que los tiempos nuevos y las nuevas tareas imponen dedicación, sacrificio y el propio perfeccionamiento, único camino para asegurar su estabilidad y porvenir (Argentina, 1933, p.22).

En el espíritu de la ley, a fines de 1937 el Directorio aprobó los fundamentos del Estatuto para el personal de la DNV, reorganizando el plantel de acuerdo con un nuevo escalafón, pautando los ascensos e instituyendo una serie de instancias de control (Argentina, 1937, p.23). El estatuto se basaba en el proyecto destinado a todos los empleados públicos nacionales, preparado por el Poder Ejecutivo y enviado al Congreso pero que no fue aprobado por dicho cuerpo. En parte, las razones del rechazo por parte del poder legislativo se basaban en que el nuevo estatuto planteaba problemas a ciertos mecanismos clientelísticos arraigados, pero fundamentalmente porque, a cambio de establecer la estabilidad del empleado público, suspendía su derecho al voto. La propuesta de Justo, de una inconstitucionalidad flagrante, extremaba en términos políticos lo que la DNV se había adelantado en implementar, señalando cuál era su verdadero norte: la construcción de una burocracia completamente ajena a la política.

Otro dato importante para examinar la relación entre el nuevo organismo y la política fue la autonomía con la cual fue dotado. Tal carácter era algo relativamente novedoso para la estructura estatal y se consideraba decisivo para que las obras se efectuaran con celeridad, ya que no requería de la aprobación de cada una de sus acciones por parte del ministro, sino que dependía de un directorio específico. Dentro del MOP ya existían algunos organismos con autonomía (Administración de Ferrocarriles del Estado y Obras Sanitarias de la Nación), aunque en el caso de la DNV se registran elementos novedosos en la forma que adoptaba esa autonomía y la manera en que era justificada. El Directorio era nombrado por el Poder Ejecutivo con acuerdo del Senado; se componía de un presidente, que representaba dentro de un cuerpo a las zonas Centro y Norte del país, vocales representantes de la zona Sur y el Litoral (dos, ingenieros funcionarios de la DNV), de las empresas de transporte (uno de los ferrocarriles), de asociaciones agrarias (uno recaería sobre la Sociedad Rural Argentina), entidades automovilísticas (uno recaería sobre el ACA) y el Ejército (un representante). En este esquema corporativo - usando el término en sentido laxo - los intereses privados, aunque en minoría, eran incorporados al organismo estatal en un directorio mixto, en el cual el control estatal se imponía desde el punto de vista numérico. En síntesis, una figura socialmente importante, asociada a la política, pero también un técnico prestigioso y en este sentido se invocaría su figura en los discursos políticos y estatales. La elección de una figura prestigiosa desde el punto de vista técnico, más allá de sus vínculos económicos y políticos, era presentada como un signo de que lo que estaba en juego no era la cesión de las decisiones estatales al campo de los intereses privados, sino una concepción de acción estatal de nuevo tipo. No era la articulación entre Estado e intereses privados lo que perturbaba a este gobierno, sino particulares inflexiones de la relación entre Estado y política.

En efecto, la presencia de empresas privadas e instituciones públicas en el Directorio era presentado como garantía de que la repartición "se mantendría alejada de la política" (Argentina, 1932, p.676). La revista del ACA - miembro del Directorio de la DNV proclamaba en 1933 que "afortunadamente puede manifestarse ahora, y así lo vienen 
confiando quienes han tenido el honor de ser los primeros dirigentes de la nueva institución, la construcción de caminos se ha emancipado de la política" (La visita..., mayo 1933). Tal tipo de expresiones se reiteraban en distintos ámbitos. En tal sentido, recordemos que 'política', en los discursos de gobernantes de los años 1930, cuando se la degradaba frecuentemente como 'politiquería', era considerada una 'mala palabra', sinónimo del gobierno del Partido Radical que en la segunda presidencia de Hipólito Irigoyen, ejercida desde 1928, había sido derrocado por el golpe militar de septiembre de 1930. El gobierno depuesto era emblematizado por sus opositores como corrupto, ineficiente y clientelista. La oposición a esta imagen de la 'política' que hacía referencia a un régimen denostado en su vinculación con el Estado, guió numerosas decisiones del nuevo organismo. De esta manera, la repartición era entendida como el modelo de un aparato estatal administrador, moderno y eficiente, profundamente diferente del heredado. Por supuesto, estas eran las representaciones de la política que sustentaban el discurso público estatal, aunque otro tipo de análisis saca rápidamente a la luz los puntos de continuidad que vinculaban la nueva creación con experiencias previas que vienen desde la actuación del personal técnico dentro de elencos estatales hasta las semejanzas entre la Ley de Vialidad de 1932 y el proyecto del mismo asunto presentado al Congreso por el gobierno radical de Marcelo T. de Alvear en 1925. Es necesario remarcar también el carácter de representaciones de estas afirmaciones, pensando en una figura como la de Allende Posse, vinculado a la política, con sus propios intereses económicos y sus vinculaciones políticas, pero presentado como un técnico, por lo tanto 'apolítico' en términos de la concepción degradada de la política.

Lo importante para nuestro análisis consiste en destacar que este discurso público operaba en la construcción de políticas estatales y que los ingenieros, en tanto técnicos avalados por un saber específico que hablaba de racionalidad, de eficiencia y precisión ocupaban el lugar de una política entendida en términos degradados: la DNV en el momento de su creación, encarnaba una renovada propuesta de acción estatal.

\section{Tensiones entre técnica y política}

¿Qué implicancias tenía esta centralidad de los ingenieros que se observa en las representaciones? ¿Cuáles eran sus límites? En primer lugar, en relación con la política, si observamos las máximas conducciones del organismo, con dos breves excepciones en 1906 y 1907 - Miguel Tedín y Carlos Maschwitz - no hubo ministros ingenieros. El ministro impulsor de la vialidad en los años 1930, Manuel Alvarado, era un político salteño, comerciante en su actividad privada, que sólo contaba con estudios universitarios incompletos en abogacía (Diccionario..., 1948). Aunque las instituciones de los ingenieros tempranamente insistieron en demandar que la conducción de estos organismos fuera ejercida por profesionales vinculados al tema, la política continuó imponiendo sus propios valores y mecanismos: nos encontramos lejos de una 'tecnocracia', aunque muchas veces se tomaran prestados sus discursos. Además, como se ha planteado anteriormente, algunas figuras con formación técnica, como es el caso de Allende Posse, tenían también fuertes vínculos con la política. A ellos no se los puede calificar como 'técnicos' a secas, hecho que los avalaba para acceder a cargos de alto rango e implicancias políticas. Un caso similar es 
el de otro presidente de la DNV en el período 1940-1943: el ingeniero mendocino Emilio A. López Frugoni, director de Obras Públicas y ministro de Obras Públicas de Mendoza entre 1930-1931 y 1932-1935, respectivamente. Un caso de ascenso meritocrático hasta el máximo cargo en vialidad se observa, en cambio, fuera del período estudiado, en el caso del ingeniero Abel Cornejo, administrador general de Vialidad Nacional entre 1943 y 1944, que había iniciado su carrera como escribiente de la Dirección General de Puentes y Caminos en 1910 (Diccionario..., 1948).

Al mismo tiempo, al menos en la década de 1930, excepto los tres cargos de mayor rango (ministro, subsecretario y oficial mayor del MOP), los lugares superiores estaban ocupados por profesionales universitarios, en su mayor parte ingenieros, con la salvedad de un arquitecto en la Dirección General de Arquitectura, un contador en la sección contable y otros casos aislados. Es decir que la 'vida interna', el desenvolvimiento cotidiano del ministerio, puede considerarse efectivamente controlado por figuras de perfil técnico. Se trata de una dirección dada desde la formación del organismo: la ley 4560/ 1905 establecía que era necesario tener título nacional de ingeniero o arquitecto para ocupar cargos vinculados a obras públicas en la administración nacional, con excepciones que dejaban espacio para los mecanismos de la política, sobre todo en los niveles superiores.

De todas formas, el control del organismo por parte de los técnicos es también un tema que debe precisarse en sus alcances y sus límites. La programación de las obras públicas siempre estuvo atravesada por decisiones políticas que no necesariamente tenían base técnica, sobre todo porque el Congreso aprobaba por ley la realización de obras propuestas por legisladores fuera de toda planificación, que luego el MOP debía incorporar a su programación de tareas. En tal sentido podría pensarse que los profesionales técnicos controlaban el aparato interno del ministerio, es decir su estructura técnico-administrativa subordinada, pero no lograban ir mucho más allá.

Pero también se pueden rastrear los esfuerzos que hizo la burocracia para intervenir en las decisiones políticas. Debemos remarcar la constitución del Consejo Nacional de Obras Públicas (1938), en la década de 1930, integrado por directores nacionales y generales (del MOP), administradores nacionales y presidido por el ministro, con amplias funciones sobre los planes orgánicos de obras públicas. Esto es lo más lejos a lo que se llegó en cuanto a que la estructura del MOP tuviera poder sobre la decisión de las prioridades de obras y no parece haber sido desdeñable (Argentina, jul. 1938, p.149). El Consejo se constituyó en circunstancias que le asignarán un poder importante: la falta de materiales y equipos importados y los aumentos de costos que acompañan los últimos años de la década de 1930 y los años de la Segunda Guerra Mundial. En efecto, se trataba de un momento que exigía reprogramar obras y prioridades en base a cierta racionalidad técnica; era necesario entonces administrar la escasez, sopesar recursos y anticiparse a posibles desbordes. Un momento ideal para estimular la planificación técnica y ratificar la necesidad de intervención del Estado, legitimando un rol que había ido adquiriendo a lo largo de la década.

Sin embargo, estos avances relativos de las decisiones técnicas sobre las políticas en algunos aspectos de la planificación de la obra pública convivían, en los años 1930 con otros datos que complejizaban el vínculo. En tal sentido, debemos considerar ante todo que no todas las representaciones creadas por el Estado alrededor de la ingeniería y de los 
ingenieros en el período se verificaban en la práctica. Ya se ha hecho referencia, aún en el caso de profesionales vinculados al Estado, al registro de un ejercicio profesional mucho más amplio y complejo que la exclusiva carrera meritocrática dentro del mismo. Otros elementos, como la de una supuesta dedicación sacrificada y orgullosa del servicio estatal, contrastan con observaciones sobre las formas concretas de ejercicio profesional dentro del Estado y con las reacciones que generaba, sobre todo acercándonos a fines de la década. Así, según afirmaba la revista La Ingeniería en 1937, la limitación de sueldos impuesta por ley en la DNV (8\% de su presupuesto) creaba diferencias con los salarios más altos que cobraban los profesionales en otras reparticiones estatales (Las remuneraciones..., 1937, p.284). La defensa frente a esta situación fue propuesta por dos diputados ingenieros, en la ley de presupuesto 1937, estableciendo el sueldo mínimo que deberían percibir los ingenieros, arquitectos y agrimensores al servicio del Estado nacional, hecho que el CAI festejaba (La demostración..., 1937, p.421). Estos datos contrastan con algunos aspectos heroicos de las representaciones y muestran miradas menos optimistas sobre las formas de ejercicio profesional de los ingenieros dentro del Estado, quienes:

No contando con capitales, ofrecen sus servicios a las reparticiones públicas, mediante ... un sueldo mensual. Cuando consideran que su experiencia es suficiente, ellos suelen ingresar en la industria privada pues ésta les brinda mejores oportunidades. En ese momento se produce la selección. Algunos permanecen en su cargo por razones personales o circunstancialmente atraídos por la estabilidad del empleo público y el aliciente de la jubilación ... El Estado pierde de esta forma a sus mejores servidores (Las remuneraciones..., 1937, p.283).

Son varias las observaciones que se pueden hacer a este texto: en este momento en el cual aún se mantenían representaciones 'estadocéntricas' de la ingeniería, la industria privada era presentada como más atractiva que el ejercicio público; en el Estado se adquiría experiencia, que luego podía ser puesta al servicio de la actividad privada; el Estado ofrecía como ventajas particulares cierta estabilidad y la jubilación. Nos enfrentamos a un cálculo tan desapasionado como poco heroico de ventajas y límites. En los años 1930, entonces, se registraban signos de que el tradicional prestigio del vínculo entre ingeniería y Estado comenzaba a verse corroído. De hecho, una serie de especialidades de la ingeniería que se desarrollan en la entreguerras, como la ingeniería industrial, que recibió particular estímulo al promoverse como carrera de la Universidad de Buenos Aires en 1920, se dirigían más a la producción privada que al campo estatal (Lobato, ago.-dic. 1998). Cuando, en las páginas de La Ingeniería, el ingeniero Julio Vela Huergo se refería en 1934 a "la hora de los ingenieros", hacía hincapié en su capacidad organizativa de la industria y el trabajo (citado en Lobato, 1998, ago.-dic. 1998, p.64).

Más allá de estos campos de acción relativamente nuevos y altamente valorados en el plano de las representaciones, otros campos tradicionales ganaban terreno en las prácticas. El desarrollo de temas de arquitectura (vivienda, sobre todo), por ejemplo, no demasiado valorado en las representaciones sobre las jerarquías de las actividades de los ingenieros, era un campo que en las décadas de 1920 y 1930 había ido creciendo en la práctica, en un rango de tamaño de obras muy amplio y que se volcaba en gran medida en el mercado privado y en el ejercicio liberal de la profesión. Los ingenieros competían con arquitectos y técnicos de formación secundaria por los proyectos, pero también tenían posibilidades de ensayar la construcción sin grandes recursos de tecnología o equipo. 
Al mismo tiempo, la propia actividad de la DNV creaba un posible campo de trabajo para ingenieros devenidos empresarios sin contar con excesivos capitales. Tal como fueron planteados, los caminos en Argentina permitían que pequeñas empresas de construcción intervinieran en licitaciones y obras. En 1936 se constituyó la Cámara de la Construcción del CAI, que años más tarde se convertirá en la Cámara Argentina de la Construcción, a secas, que se originó en la convocatoria de quince empresas dirigidas o propiedad de ingenieros, dentro de la asociación que los representaba. No eran las empresas de la construcción más importantes, pero para 1945 ya se había transformado en el órgano corporativo de propietarios de empresas más importante y en un interlocutor privilegiado para el Estado.

Las relaciones precisas entre estos distintos datos que marcan el vigor de nuevos campos de desarrollo profesional de los ingenieros y de nuevas corrientes científicas dentro de la ingeniería exigen de estudios más precisos que exceden los límites de este trabajo. En el marco de este análisis son presentados simplemente como indicaciones de que la fuerte relación con el Estado de décadas anteriores, sin desaparecer, va siendo desplazada por nuevos intereses, que se registran tanto en el plano de las prácticas como en el de las representaciones generadas por el campo de la ingeniería.

Sin embargo, las rupturas más fuertes entre las instituciones representativas de los ingenieros y el Estado se produjeron en la competencia con la política por la dirección del Estado, a partir del golpe militar de 1943, agudizándose durante el peronismo (Healey, 2000; Ballent, 2005b). Como lo había hecho en 1930, el golpe militar invocó una crítica a la política en su sentido tradicional, pero esta vez no apeló a la figura de los ingenieros como moralizadores, sino que se buscó las virtudes del militar. En rigor, sobre todo por su concentración en el MOP, los ingenieros fueron atacados como representantes de un Estado inoperante o permeado por intereses privados. El Consejo de Obras Públicas, que otorgaba poder a los técnicos, fue disuelto. Las reparticiones con autonomía, que anteriormente habían sido consideradas instituciones ejemplares, perdieron tal carácter y su acción fue cuestionada por la nueva conducción militar. En 1945 se despidieron profesionales y se sucedieron renuncias; el CAI asumió la defensa de los profesionales y de las reparticiones, considerando que el propio honor y prestigio de la ingeniería argentina estaba puesto en juego. Según el presidente del CAI, "la ingeniería (había sido) desplazada de la función técnico directiva que le corresponde ejercer por su naturaleza específica" (Defensa de la ingeniería..., 1945, p.154; Lucchini, 1981, p.428-442).

En rigor, atendiendo a la historia del vínculo entre ingeniería y MOP, lo que se registra es la irrupción de un tipo de política que no respetaba la lógica con la cual la institución se había creado y consolidado. Una etapa de la historia del organismo comenzaba a cerrarse. Su estructura se mantendría, aun durante el peronismo, pero en una relación con la política muy diferente y habiendo roto las relaciones con las instituciones de los ingenieros, incluida la Facultad de Ciencias Exactas, transformada en 1952 en Facultad de Ingeniería. Con la caída del peronismo, muchos de los ingenieros actuantes en los años 1930 volvieron al MOP. En tal momento se intentó también restaurar el prestigio de sus antiguas reparticiones, las 'glorias pasadas' parecían ser modelos capaces de iluminar el futuro. En este marco, Allende Posse volvió a ser nombrado al frente de Vialidad Nacional entre 1956 y 1958. Sin embargo, el nuevo contexto era ya profundamente diferente tanto en lo político como en 
lo técnico y en cuanto a la forma y funciones del aparato estatal. En este nuevo contexto, la relación ingeniería-Estado ya no ocuparía el rol privilegiado cuya cima hemos observado en los años 1930.

\section{Conclusiones}

El artículo ha tomado como centro la creación de una institución estatal de gran relevancia en las transformaciones territoriales argentinas, en un período también clave de ingerencia del Estado en el sector. Se trató de una institución, que, en el período inicial de su desarrollo, en líneas generales y según las fuentes del período, parece haber cumplido con las expectativas de la época (Cómo se han cumplido..., 1940). Pese a ello, la acción no dejó de mostrar debilidades y conflictos, que la historiografía ha señalado, como el trazado de la red troncal de caminos en paralelo con las vías de los antiguos ferrocarriles (García Heras, 1985). El tema es complejo y la evaluación precisa de su impacto supera los alcances de este trabajo, pese a lo cual múltiples fuentes de la época nos permiten rescatar la representación del momento que registraba a la DNV como una institución prestigiosa, mientras que la actual estructuración troncal del sistema vial argentino mantiene el trazado proyectado en los años 1930. La acción del Estado resultó en este caso perdurable.

En particular, hemos apelado al estudio de la repartición para señalar el vigor de un vínculo: el de la ingeniería y el Estado. La DNV se encontraría entonces en un momento de culminación de ese proceso, que se registra desde fines del siglo XIX, a partir de la formación del MOP, contemporáneo o algo posterior a la constitución de otras instituciones del campo científico y profesional de los ingenieros. Lo que observamos en los años 1930, entonces, sería el resultado de una historia, actualizada por las particulares circunstancias políticas del período 1930-1943 en Argentina.

El trabajo se ha detenido en las representaciones de las trayectorias profesionales de ingenieros, elaboradas por el Estado, coincidentes con las de las instituciones de los ingenieros, para señalar el carácter que puede calificarse de 'estadocéntrico' de las mismas, en las cuales se privilegia la pertenencia al Estado y el ascenso meritocrático. Se han relacionado estas representaciones con el rol del MOP en el campo disciplinar y profesional de la ingeniería en Argentina y con la consolidación de una burocracia técnica, donde las figuras técnicas, aunque no alcanzaran los más altos lugares directivos dentro del MOP, tenían un lugar destacado y ganaban posiciones en el control de la obra pública como lo demostró en 1938 la constitución del Consejo de Obras Públicas, que operó hasta 1944.

Al mismo tiempo, el artículo ha registrado en el período, sobre todo hacia fines de la década, una serie de datos de distinta procedencia dentro del campo de la ingeniería que informan del crecimiento de nuevas ramas de la ingeniería ligadas a la empresa privada, de representaciones producidas por los ingenieros que comienzan a poner en duda el valor de la relación profesional con el Estado y de la importancia creciente de los ingenierosempresarios de la construcción, cosa que se observa en la creación del Cámara Argentina de la Construcción del CAI en 1936.

Si esos datos hacen referencia a procesos lentos y de naturaleza distinta aunque convergentes para el tema que nos ocupa, un conflicto en gran medida político instauró 
una ruptura en el vínculo entre ingeniería y Estado. Tales episodios han sido estudiados por otros trabajos, pero a la luz del análisis presente se afirma que lo que se presenta como un conflicto político (o lo que deviene en conflicto político), se forja, en rigor, en el conflicto planteado por la forma asumida por la constitución del vínculo entre ingeniería y Estado y la aplicación de una nueva lógica en una estructura consolidada. En tanto esta situación tiñe más de una década de desarrollo del ministerio, el intento de restauración de un vínculo y de un modelo de gestión después de la caída del peronismo resulta ya imposible. En 1943, según nuestra hipótesis, se ha cerrado una etapa institucional iniciada a fines del siglo XIX: la del programa inicial del MOP y la construcción de su burocracia técnica de ingenieros.

Este trabajo reconoce su carácter preliminar y la necesidad de profundizar en buena parte de los temas abordados, así como la posible construcción de nuevas hipótesis para proseguir los estudios. En tal sentido, todo el derrotero que hemos señalado sugiere como campo de estudio el de un grupo profesional que puede usar su experiencia colectiva en la construcción del Estado para interpelar a ese mismo Estado desde la acción privada.

\section{REFERENCIAS}

ARGENTINA.

Cámara de Senadores. Diario de Sesiones, Buenos Aires, p.65. 16 mayo 1939.

ARGENTINA.

Ministerio de Obras Públicas. Dirección

Nacional de Vialidad. Memoria 1938. Buenos Aires: Ministerio de Obras Públicas. 1939.

\section{ARGENTINA.}

Decreto del 24 jun. 1938. Obras Públicas y Privadas. Buenos Aires: s.n. p.149-151. jul. 1938.

ARGENTINA.

Poder Ejecutivo Nacional. Obra de gobierno 1932-1938. v.2. Buenos Aires: s.n. 1938.

\section{ARGENTINA.}

Ministerio de Obras Públicas. Dirección Nacional de Vialidad. Memoria 1937. Buenos Aires: Ministerio de Obras Públicas. 1937.

\section{ARGENTINA.}

Ministerio de Obras Públicas. Dirección Nacional de Vialidad. Conferencia pronunciada por el ingeniero Justiniano Allende Posse al regreso de su viaje de estudios a Sur y Norte América. Buenos Aires: Ministerio de Obras Públicas. 1936.

\section{ARGENTINA.}

Ministerio de Obras Públicas. Dirección Nacional de Vialidad. Memoria 1935. Buenos Aires: Ministerio de Obras Públicas. 1935.

\section{ARGENTINA.}

Ministerio de Obras Públicas. Dirección Nacional de Vialidad. Discurso pronunciado por el ingeniero Justiniano Allende Posse inaugurando el ciclo de Conferencias sobre Técnica de Caminos el 3 de agosto de 1934. Buenos Aires: Ministerio de Obras Públicas. 1934a.

\section{ARGENTINA.}

Ministerio de Obras Públicas. Dirección Nacional de Vialidad. Memoria 1934. Buenos Aires: Ministerio de Obras Públicas. 1934b.

\section{ARGENTINA.}

Ministerio de Obras Públicas. Dirección Nacional de Vialidad. Memoria 1933. t.1. Buenos Aires: Ministerio de Obras Públicas. 1933.

\section{ARGENTINA.}

Cámara de Diputados. Diario de Sesiones, Buenos Aires, p.676. 1932.

BALLENT, Anahi.

Kilómetro cero: la construcción del universo simbólico del camino en la Argentina de los años treinta. Boletín del Instituto de Historia Argentina y Americana Dr. Emilio Ravignani, n.27, p.107-137. 2005a.

BALLENT, Anahi.

Las huellas de la política. Vivienda, ciudad, peronismo en Buenos Aires, 1943-1955. Buenos Aires: Universidad Nacional de QuilmesPrometeo. 2005b.

BALLENT, Anahi. La red nacional de caminos y la construcción de lugares turísticos en la década de 1930. Ponencia presentada al Taller Internacional 
Desplazamientos, Lugares, Identidades, 11-13 mayo 2004. Buenos Aires. 2004.

BALLENT, Anahi et al. Ministerio de Obras Públicas de la Nación. In: Liernur, J.F. et al. (Ed.). Diccionario de arquitectura en la Argentina. t.I-N. Buenos Aires: Clarín. 2004

BALLENT, Anahi et al.

País urbano o país rural: la modernización territorial y su crisis. In: Cattaruzza, A. (Dir. de tomo). Nueva Historia Argentina. Tomo 7: Crisis económica, avance del Estado e incertidumbre política. Buenos Aires: Sudamericana. p.143-200. 2001.

BARBERO, María I. et al. (Ed.). Americanización: Estados Unidos y América latina en el siglo XX. Buenos Aires: Eduntref. 2003.

BESIO MORENO, Nicolás.

Galería de ingenieros argentinos eminentes ya desaparecidos. La Ingeniería, Buenos Aires, n.843, p.50-67. ene. 1945.

CAMINOS...

Caminos a construirse en 1933 y obras en ejecución. Automovilismo, Buenos Aires, n.167, mayo 1933.

CÓMO SE HAN CUMPLIDO..

Cómo se han cumplido los primeros objetivos de la vialidad argentina. La Ingeniería, Buenos Aires, p.548-561. 1940.

DEFENSA DE LA INGENIERÍA...

Defensa de la ingeniería y de los ingenieros argentinos por el CAI. La Ingeniería, Buenos Aires, p.154. 1945.

\section{DICCIONARIO...}

Diccionario biográfico contemporáneo: personalidades de la Argentina. Buenos Aires: Editorial Veritas. 1948.

ESPÓSITO, Juan.

Organización del Ministerio de Obras Públicas de la Nación. Revista de Obras Públicas y Privadas de la República Argentina, Buenos Aires, n.86. 1944.

GARCÍA HERAS, Raúl.

Automotores norteamericanos, caminos y modernización urbana en la Argentina, 1918-1939. Buenos Aires: Libros de Hispamérica. 1985.

GORELIK, Adrián.

La arquitectura de YPF, 1934-1943: notas para la interpretación de las relaciones entre Estado, modernidad e identidad en la arquitectura argentina de los años treinta. Anales del Instituto de Arte Americano, Buenos Aires, n.25, p.97-106. 1987.
HEALEY, Mark.

The ruins of the new Argentine: peronism, architecture and the remaking of San Juan alter the 1944 earthquake. Dissertarion (PhD) Department of History, Duke University, Durham. 2000

HUMET, Enrique.

Caminos económicos. Interesantes observaciones del ingeniero jefe de la Sección Caminos de Costo intermedio y Bajo Costo de la Dirección Nacional de Vialidad. Automovilismo, Buenos Aires, n.201, jul. 1936.

KOROL, Juan C.

La economía. In: Cattaruzza. A. (Dir. de tomo). Nueva Historia Argentina. Tomo 7: Crisis económica, avance del Estado e incertidumbre política. Buenos Aires: Sudamericana. p.17-47. 2001.

LA DEMOSTRACIÓN...

La demostración a los diputados nacionales ingenieros Julio A. Noble y Herminio Arrieta. $\mathrm{La}$ Ingeniería, Buenos Aires, p.421. 1937.

LAS REMUNERACIONES...

Las remuneraciones del personal de la Dirección Nacional de Vialidad. Trabajo presentado al tercer Congreso Argentino de Vialidad por representantes del Centro Argentino de Ingenieros'. La Ingeniería, Buenos Aires, p.282285. 1937.

LA VISITA...

La visita del señor presidente a las canteras de Tandil y Olavarría. Automovilismo, Buenos Aires, n.167, mayo 1933.

LOBATO, Mirta.

La Ingeniería: industria y organización del trabajo en la Argentina de entreguerras. Estudios del Trabajo, Buenos Aires, n.16, p.47-68. ago.-dic. 1998.

LOS MATERIALES...

Los materiales argentinos para construir caminos. Automovilismo, Buenos Aires, n.167, mayo 1933.

LUCCHINI, Alberto P.

Historia de la ingeniería argentina. Buenos Aires: Centro Argentino de Ingenieros. 1981.

MACOR, Darío.

Partidos, coaliciones y sistema de poder. In: Cattaruzza, A. (Dir. de tomo). Nueva historia argentina. Tomo 7: Crisis económica, avance del Estado e incertidumbre política. Buenos Aires: Sudamericana. p.49-95. 2001.

OSPITAL, María Silvia.

Autos y caminos para la modernización de Argentina: comerciantes importadores de automóviles, 1920-1940. In: Jornadas de 
Historia Económica, 18., 18-20 sept. 2002, Mendoza. In: Actas del... Versión CD. 2002.

OSZLAK, Oscar.

La formación del Estado argentino: orden, progreso y organización nacional. Buenos Aires: Planeta. 1997.

PALERMO, Silvina.

Elite técnica y Estado liberal: la creación de una administración moderna en los Ferrocarriles del Estado (1870-1910). Trabajo de discusión en grupo de estudio Elites Estatales y Estudios del Estado, Instituto de Desarrollo Económico y Social, Buenos Aires. 2005.

PIGLIA, Melina.

Asociaciones civiles y Estado en los años veinte: las intervenciones públicas del Automóvil Club Argentino y del Touring Club Argentino en materia de vialidad y turismo. Artículo inédito. 2007.

PIGLIA, Melina.

Ciudades de lona: el Automóvil Club Argentino y la práctica del camping en la entreguerras (1926-1939). Ponencia presentada al Taller Internacional Desplazamientos, Lugares, Identidades, 11-13 mayo 2005. Buenos Aires. 2005.

SALERNO, Elena.

Los comienzos del Estado empresario: la Administración General de los Ferrocarriles del Estado (1910-1928). Buenos Aires: Facultad de Ciencias Económicas/Universidad de Buenos Aires. 2003.

SCARZANELLA, Eugenia.

El ocio peronista: vacaciones y turismo popular en Argentina (1943-1955). Entrepasados, Buenos Aires, n.14, p.65-86. 1998.

SILVESTRI Graciela.

El color del río: historia cultural del paisaje del Riachuelo. Buenos Aires: Universidad Nacional de Quilmes - Prometeo 2003.

TORRENT, Horacio.

Signos modernos y territorio: el elevador de granos y el paisaje del cereal. Tesis (Magíster en Arquitectura) - Pontificia Universidad Católica de Chile, Santiago de Chile. 2001. 